\title{
¿CÓMO PODEMOS GARANTIZAR LA SOSTENIBILIDAD ECONÓMICA Y AMBIENTAL DE UNO DE LOS PAÍSES CON MAYOR HUELLA ECOLÓGICA DEL MUNDO?
}

\author{
HOW CAN YOU ENSURE THE ECONOMIC AND ENVIRONMENTAL \\ SUSTAINABILITY OF ONE OF THE COUNTRIES WITH THE HIGHEST \\ ECOLOGICAL FOOTPRINT PER CAPITA IN THE WORLD?
}

\author{
Oscar Gallego Pérez \\ United World College of South East Asia, Singapur email: oga@uwcsea.edu.sg
}

Resumen

El país de los Emiratos Árabes Unidos posee un ecosistema desértico y de limitados recursos naturales. Con la mejora del nivel de vida, los Emiratos Árabes Unidos ha incrementado el gasto energético y la demanda de productos importados y otros recursos naturales. Como consecuencia, EAU se ha situado por primera vez como el país con huella ecológica per cápita más alta del mundo.

El objetivo de este proyecto es dar a conocer los problemas medioambientales que aqueja el país debido a la mala gestión de sus recursos, y crear un plan de acción que haga el sector del agua y de los residuos más eficiente y limpio con la utilización de energías renovables y de nuevas tecnologías.

Para la elaboración de este proyecto se utilizaron diferentes metodologías. Como fuentes cualitativas, se entrevistaron y visitaron empresas privadas y públicas del sector energético y como métodos cuantitativos, se realizaron cuestionarios.

Palabras clave: Sostenibilidad ambiental, recursos naturales, huella ecológica, energías renovables, residuos

\section{Abstract}

The country of the United Arab Emirates has a desert ecosystem and limited natural resources. Over the years, they have significantly increased energy expenditure and its capacity and demand for imported products and other natural resources from abroad. As a result, the UAE has placed first as the country with per capita ecological footprint highest in the world.

The objective of this project is to present the environmental problems faced by the country due to the poor management of their resources; and to create an action plan to make the water and waste sectors, cleaner and more efficient, with the use of renewable energies and new technologies.

For the development of this project different methodologies were used. As qualitative sources, I met and visited private and public companies in the energy sector and as quantitative methods, I created questionnaires.

Keywords. Environmental sustainability, footprint, waste, renewable energy,

Introducción

Emiratos Árabes Unidos en contexto - Un país en transformación: el país de Emiratos Árabes Unidos ha experimentado un largo período de extraordinario crecimiento económico debido a la capitalización de sus recursos de petróleo y gas, que a su vez ha dado lugar a un rápido desarrollo y mejores niveles de vida. Sin embargo, para sostener este desarrollo se ha estado consumiendo cantidades 
crecientes de recursos naturales como la energía, madera, alimentos y fibra (Centro de Estadística de Abu Dabi, 2011)

A pesar de que Dubái y Abu Dabi sean consideradas como las dos ciudades con mejor calidad de vida en todo el Medio Oriente y África, el desarrollo humano no ha sido distribuido de forma equitativa y ha dejado un lastre ambiental, social, económico y demográfico entre otros.

La explosión demográfica que ha sufrido los Emiratos Árabes Unidos en los últimos 15 años ha tenido consecuencias muy negativas para el medio ambiente, creando un deterioro progresivo de su biosfera.

La demanda por agua en este país con una población en crecimiento, ha hecho que las fuentes de agua natural se estén agotando. Al mermar el nivel acuífero (a un ritmo de un metro por año, desde los últimos 30 años), el agua salada del mar penetra para rellenar el espacio. Este proceso hace que el agua dulce se contamine, especialmente cerca de la costa, donde el nivel de salinidad del terreno afecta a la fertilidad de la tierra, dificultando la agricultura (Centro de Estadística de Abu Dabi, 2011)

EAU tiene actualmente el consumo más alto de agua per cápita en el mundo; cada residente utiliza una media de 550 litros por persona, por día, comparado con los 85 litros en Jordania, país vecino con un clima similar. Para poder abastecer a la demanda, plantas de desalinización de agua se han construido alrededor del país, incluyendo la mayor planta del mundo que se encuentra en Jebel Ali, Dubái; aunque solucione el problema de abastecimiento de agua, la desalinización crea nuevos problemas. Para facilitar la disponibilidad del agua, el país ha invertido fuertemente en la desalinización, produciendo en el 2010 una media de 9 millones de metros cúbicos, con un coste de 18 millones de dólares al día (Simpson, 2013). Este elevado consumo y el escaso control y ahorro por parte de las autoridades, hace que el agua tenga un coste energético altísimo, ya que las centrales desalinizadoras necesitan de una central energética que consume grandes cantidades de gas natural o fuel. Asimismo producen enormes cantidades de agua altamente salina, las cuales son vertidas al mar en detrimento de la vida marina (Centro de Estadística de Abu Dabi, 2011; Maktoob, 2015)

Desde el descubrimiento del petróleo en el Golfo Pérsico, muchos han sido los cambios en el ámbito del consumo de los habitantes de las sociedades beneficiadas. Los Emiratos han pasado en menos de 30 años de tener una sociedad prácticamente nómada y dependiente de la agricultura y pesca, a una economía capitalista, basada en el consumo.

La concentración de altos ingresos produce una demanda económica orientada hacia la producción para los sectores más ricos que incrementa el uso de los recursos naturales generando el sobre consumo, causa básica del deterioro ambiental en los países desarrollados. Consecuentemente, han aumentado las desigualdades en la sociedad con la implantación del nuevo modelo basado en el consumismo.

Según WWF (World Wide Fund for Nature) Living Planet Report, EAU fue en 2007 el país con el mayor nivel de impacto ecológico por persona del mundo, y desde entonces se ha mantenido en los primeros puestos. Los niveles de consumo de energía y agua son también de los más elevados mundialmente, y sus residentes son de los que más basura producen (Emirates News Agency, Wam, 2014a,b). .

Las imponentes construcciones de proyectos en las costas del país, tales como Palm Islands y Dubái Waterfront, destruirán los arrecifes de corales y los bancos de peces, así como un daño irreparable en los lugares de cría de especies en peligro de extinción tal como la tortuga carey. De forma similar, las grandes construcciones del desierto, como es el caso de Dubailand, tendrá un impacto no sólo en el paisaje, pero en la fauna y flora del desierto.

El impacto del enorme desarrollo tecnológico sufrido en los últimos años en EAU y sus consecuencias tanto positivas, como negativas están contribuyendo a la crisis ambiental planetaria.

El boom de la construcción en ciudades como Dubái, que ha ostentando durante años el título de tener una cuarta parte de todas las grúas de construcción del mundo, ha creado problemas medioambientales, entre otros la contaminación acústica y de aire, problemas de tráfico y edificios densamente poblados, provocando niveles muy altos de emisiones de gases de efecto invernadero. La contaminación doméstica, el incremento descontrolado de vertederos y su sedimentación, la caza abusiva, la pesca excesiva, una agricultura insostenible, y la eutrofización son problemas que se han 
agravado debido a una mala administración y gestión del desarrollo tecnológico (Environment Agency 2013, 2014; Emirates News Agency, Wam, 2014a,b).

Los Emiratos Árabes Unidos tiene un marco institucional federal en cada uno de los 7 emiratos que compone el país. Esta responsabilidad institucional es relativamente nueva y está en un proceso de evolución y desarrollo. Cada emirato es gobernado por una familia, en el que el soberano o gobernante puede hacer decretos de ley, estos ocasionalmente conciernen a temas medioambientales como el establecimiento de aéreas protegidas o la prohibición de caza en un emirato (Environment Agency 2013, 2014; Emirates News Agency, Wam, 2014a,b).

Debido a su enclave geográfico, EAU ha sufrido un incremento de migraciones internacionales, especialmente por razones ambientales, como es el caso de ciudadanos pakistanís, por el reciente problema de las inundaciones en este país vecino, o de habitantes de Sri Lanka, India o Indonesia que emigraron a los Emiratos después de la catástrofe del tsunami en 2004. Las revueltas populares en los últimos años de numerosos países árabes, ha hecho que Dubái se convierta en un destino popular para buscar no sólo trabajo y estabilidad económica sino el futuro de una generación de jóvenes sirios y egipcios (Centro de Estadística de Abu Dabi, 2011; Heroes of the UAE, 2015; Instituto de Recursos Mundiales, 2015).

Quizás el resultado de vivir en una nación habitada en su mayoría por expatriados, haga que el residente medio de EAU sienta tener poca responsabilidad hacia el cuidado del medio ambiente del país.

Además a pesar de la enmascarada seguridad del país, no debemos olvidar la globalización del terrorismo y la amenaza de conflictos bélicos de graves consecuencias ambientales, EAU debe ser cautelar por su situación territorial. La inestabilidad política, del país vecino Irán, con otros países como Israel o Estados Unidos, hace que la alarma de una guerra nuclear esté en el pensamiento de todos pero en boca de nadie (Centro de Estadística de Abu Dabi, 2011).

Aparte de los suministros de energía y el sol, existen muy pocos recursos naturales. La alta huella ecológica indica que se está consumiendo más recursos naturales de lo que el planeta puede regenerar naturalmente. Y si se sigue consumiendo sin cuidado ni responsabilidad, sólo puede haber una conclusión: que un día se acabe.

\section{PROBLEMÁTICA}

Justificación

Aparte de los suministros de energía y el sol, existen muy pocos recursos naturales. La alta huella ecológica indica que se está consumiendo más recursos naturales de lo que el planeta puede regenerar naturalmente. Y si se sigue consumiendo sin cuidado ni responsabilidad, sólo puede haber una conclusión: que un día se acabe.

Ha empezado una nueva etapa en la economía del país. Hace 30 años, EAU, un estado relativamente pequeño con una población nómada, alardeaba de una de las grandes reservas de crudo del mundo. Sin embargo, el despilfarro energético de las últimas dos décadas ha dejado al emirato de Dubái sin petróleo, con reservas mínimas de gas y dependiente del turismo. Ahora es el mejor momento de iniciar una estricta política ambiental nacional que involucre a todos los sectores y a los ciudadanos.

Preguntas

¿Puede el país de EAU mantener el ritmo de consumo energético y de agua?

¿Por qué se utilizan casi exclusivamente energías no renovables, como el petróleo y gas, para la producción de electricidad?

¿Qué energías renovables son posibles explotar en el país y cuáles son las que el gobierno va a introducir en los próximos 15 años?

¿Son los subsidios energéticos y del agua sostenibles a largo plazo?

¿Es positivo para los ciudadanos recibir subsidios?

Objetivos

Objetivo general 
El objetivo de este proyecto es dar a conocer los problemas medioambientales que aqueja el país debido a la mala gestión de sus recursos y encontrar medidas en el que los ciudadanos sean partícipes de la sostenibilidad económica y ambiental de los Emiratos Árabes Unidos.

\section{Metodología de Investigación}

Para la elaboración de esta investigación se utilizaron diferentes metodologías y fuentes de información. Como métodos cualitativos se realizaron entrevistas estructuradas y no estructuradas, grupos de enfoque, observaciones, visitas y contacto electrónico a empresas públicas del sector energético en los emiratos de Dubai, Abu Dabi y los emiratos del norte (Dewa, Tadweer, Masdar, Abu Dhabi National Energy Company y Horizon Energy entre otras) para generar datos descriptivos. Asimismo se acordaron entrevistas estructuradas y no estructuras y contacto electrónico a instituciones y empresas públicas y privadas de los sectores del agua y de la gestión de residuos en los siete emiratos (Beeah, Averda, Enviroserve, Zenath, Corodex, Abu Dhabi Water \& Electricity Authoroty y United Arab Emirates Ministry of Environment \& Water entre otras), aunque destacaron los emiratos de Dubai, Abu Dabi y Sharjah por ser los que poseen mayor concentración de empresas de ésta índole.

La metodología cuantitativa de este estudio se realizó mediante la recolección de datos a través de cuestionarios. Se utilizaron dos medios de recogida de datos: cuestionarios en papel y cuestionarios en línea.

Los cuestionarios en papel se distribuyeron en las playas, en los centros comerciales (Abu Dhabi Mall, Al Raha Mall, Al Wadah Mall, Khalidiya Mall, Madinat Zayed Shopping Mall, Dubai Mall, Emirates Mall, Al Ain Mall, Bawadi Mall, Sharjah City Centre), parques públicos, y universidades (Zayed university, American University of Sharjah, Fujairah College, City University College of Ajman, Abu Dhabi University).

El enlace a los cuestionarios en línea fue enviado a los empleados de una serie de empresas (Tamweel, National Bank of Umm Al Qaiwain, Ajman Bank, Deyaar Development Company, Danagas Co., Aramex, Etisalat), a escuelas públicas e internacionales y miembros, tanto empleados como estudiantes, del Instituto Masdar de Ciencia y Tecnología de la Universidad Zayed. La encuesta de papel y la encuesta en línea fueron equivalentes en términos de contenido. Todos los datos se recogieron en los siete emiratos durante un período de tres meses entre junio y agosto de 2015 . Alrededor del $89 \%$ de los datos se recogieron con la encuesta de papel y alrededor del $11 \%$ se recogió mediante la encuesta en línea. Los cuestionarios fueron traducidos a tres idiomas: inglés, árabe e hindi.

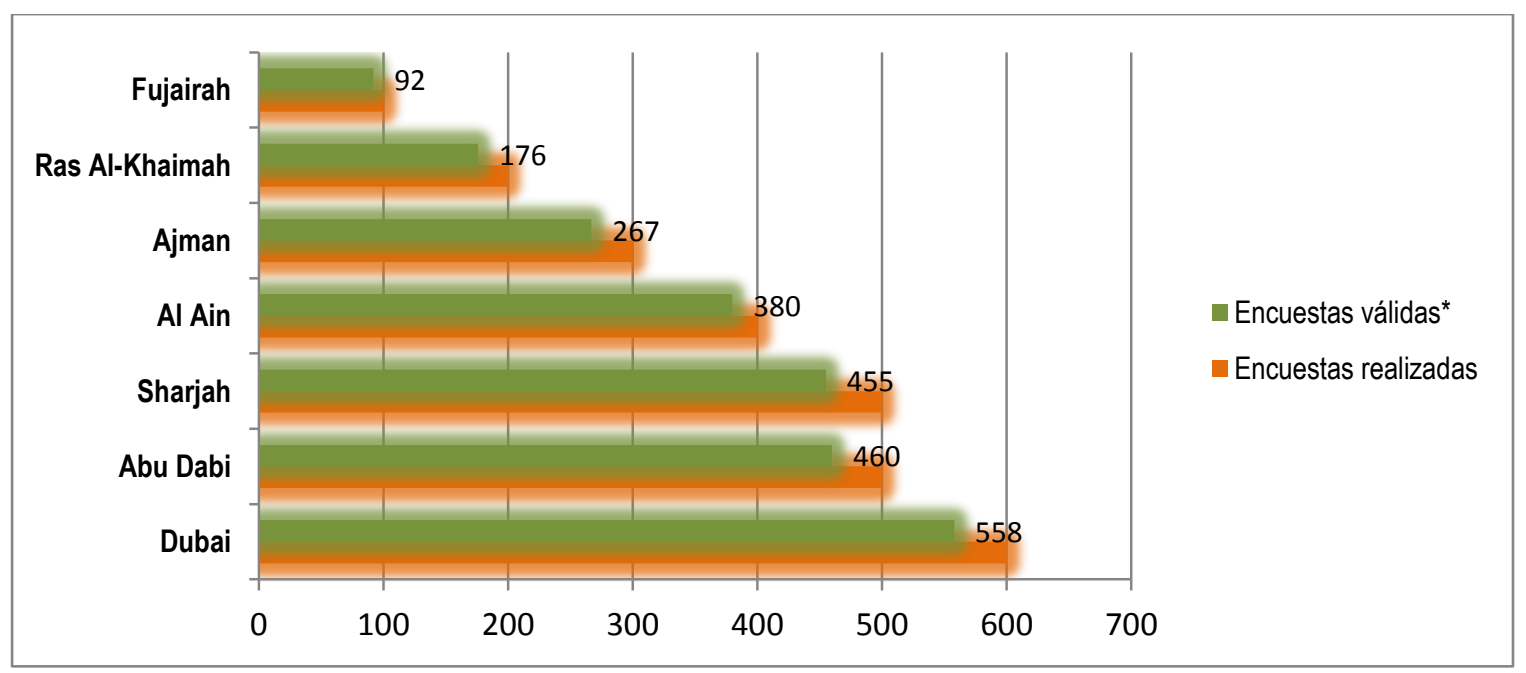

Figura 1. Cuestionarios realizados por ciudad entre junio y agosto de 2015 
Como fuentes secundarias, he utilizado métodos de consulta e investigación en publicaciones especializadas, informes y bases de datos de compañías y literatura de estudios anteriores relacionadas con esta investigación (Ojeda, 2011).

Resultados

El $80 \%$ de la huella de carbono de los Emiratos Árabes Unidos procede de la generación de agua y energía y el $70 \%$ de la energía generada va directamente a los aparatos de aire acondicionado.

Para poder responder a la pregunta hay que indagar en el sector del agua y de los residuos del país así como el consumo energético.

Los residuos

El progreso y el rápido crecimiento económico del país, además de un aumento de la población, ha dado lugar a la aparición de problemas ambientales urbanos. Esto ha llevado a un aumento constante de la proporción de los residuos generados en el país que alcanza a 1,82 kilogramos (Al Raisi, Com. Pers.) per cápita en 2014.

Como resultado de la falta de infraestructuras, los flujos de residuos se mezclan y se convierten en inservibles para el reciclado lo cual influye en el que la mayoría de los residuos se envíen a vertederos. El vertedero más grande del país es el vertedero sanitario de Al Dhafra con una superficie de casi 1.000 hectáreas, se estima que hay entre 60 y 90 millones de toneladas de residuos mezclados depositados en este vertedero.

El reciclaje en EAU está muy por debajo de su potencial y se están desperdiciando grandes cantidades de recursos valiosos mediante el envío de residuos al vertedero. En Abu Dabi, el emirato que más recicla, sólo el 36\% de los residuos se recuperó en 2011 , cifra que se acerca a la media europea, que está actualmente en el 42\%. Sin embargo, EAU con una tasa de reciclaje del $29 \%$ en 2014 , está muy lejos de países como Austria (63\%), Alemania (\% 62\%), Bélgica (58\%), Holanda (51\%) y España, que en 2014 recicló el 73,3\% de los residuos domésticos (Ecoembes).

La quema de residuos ha contribuido a la contaminación del aire, y la descomposición natural de las sustancias orgánicas en las capas inferiores del vertedero produce emisiones de metano altamente inflamables. Los departamentos de salud pública de los emiratos de Fujairah, Ras Al Khaimah, Ajman, Umm Al Quwain y Sharjah están buscando alternativas a los vertederos tradicionales, sobre todo cerca de las áreas residenciales. En junio de 2015 sólo había un vertedero tradicional en el emirato de Ajman, dos en Umm Al Quwain y tres en Ras Al Khaimah. El emirato de Fujairah tenía 11 en 2011, pero ha reducido este número a ocho en los últimos cuatro años. Ahmed Al Hammadi, director del departamento de obras y servicios públicos en Ras Al Khaimah, comentó el proyecto que el Ministerio del Medio Ambiente y Agua tiene para la eliminación segura de los residuos peligrosos a finales del 2015. Los 40 vertederos de los Emiratos del Norte se combinarán en una sola parcela, como parte de un ambicioso plan de gestión de residuos para deshacerse de todos los vertederos tradicionales, donde los residuos reciclables se harán in situ, los orgánicos se enviarán a una fábrica de fertilizantes, y el resto se enterrará en vez de quemarse (Al Hammadi, A., com. pers.).

Sin embargo, tal y como se observa en la gráfica 2, el porcentaje de la población que separa los materiales reciclables en casa para su después entrega en los puntos de reciclaje es realmente bajo. Más de la mitad de la población en los 7 emiratos reconoce no reciclar nunca o hacerlo en raras ocasiones, tan solo un tercio de los residentes de los Emiratos Árabes Unidos recicla con asiduidad.

Los Emiratos Árabes Unidos tiene como objetivo estar entre los países líderes en la gestión de residuos para el año 2030. Para que ello suceda se necesita invertir en nuevas infraestructuras para el tratamiento de residuos, una valorización y eliminación exhaustiva de los materiales, actualizar el marco jurídico y la centralización de las administraciones, así como incentivar y sensibilizar a los consumidores y a los productores sobre las ventajas de reducir, reutilizar y reciclar los residuos.

La energía

Aunque los Emiratos Árabes Unidos tenga más de un 8 por ciento de las reservas probadas de petróleo del mundo, el país sigue adelante con varios proyectos para reducir la dependencia de los combustibles fósiles. Abu Dabi quiere generar un 7 por ciento de sus necesidades energéticas totales de las energías renovables para el año 2020 y ha creado la firma verde Masdar para llevar a cabo proyectos 
que implementen su estrategia verde. Dubái quiere producir el 5 por ciento de su energía basada en energía solar para el año 2030. Pero estos proyectos pueden verse en peligro por cualquier descenso en los precios del petróleo, que tendrá un impacto para las empresas de energías renovables cuyas acciones han sido afectadas en los últimos meses. Las inversiones en energías limpias cayeron a 251.000 millones de dólares en 2014 de un máximo de 317.000 millones de dólares en 2011 (Saadi, D., 2014).

Los Emiratos Árabes Unidos, con la segunda economía árabe más grande, desarrollará una cartera energética diversificada y eficiente que sostendrá el crecimiento económico futuro y extenderá la posición del país como un proveedor estable de petróleo crudo a los mercados mundiales.

La demanda de electricidad en los Emiratos Árabes Unidos, que se genera casi exclusivamente de las plantas de energía de gas natural, se estima que aumente a un ritmo de alrededor del 9 por ciento anual hasta el año 2020. El aumento de la demanda de gas de las centrales eléctricas y de la industria petroquímica y de acero, ha convertido a los Emiratos Árabes Unidos en un importador neto de gas en la última década, lo que está provocando que desde el 2014 el gobierno empiece a pensar en la diversificación energética y en inversiones multimillonarias en el desarrollo de la energía nuclear y en menor medida de las energías renovables.

Hoy en día, Asia es el principal consumidor de hidrocarburos del Golfo, países como India, China, Japón y Corea del Sur consumen hasta un 70 por ciento del crudo procedente del Golfo. Esta dependencia energética va a crecer, y con ella la necesidad de una mayor cooperación entre los consumidores asiáticos y los productores de hidrocarburos en el Golf.

En la actualidad todas las plantas de electricidad en Dubái funcionan con gas, pero en 2030 Dubái tiene previsto llegar al 5 por ciento de sus necesidades energéticas a partir de energía solar, el carbón proporcionará el 12 por ciento, la energía nuclear el 12 por ciento y el resto provendrá del gas. Dubái tiene la intención de obtener la energía nuclear, de Abu Dabi, donde las autoridades están construyendo una planta nuclear. La demanda de energía en Dubái creció en un 5 por ciento en 2014 y la capacidad instalada actual es de 9.700 MW Los Emiratos Árabes Unidos en su conjunto tendrá que generar 160.000 puestos de trabajo en 2030 para alcanzar sus objetivos de economía verde (Carvalho S. \& El Dahan 2015).

Aunque su "propuesta de economía" verde es cuestionable, un país que tiene 360 días de sol al año que tenga como objetivo producir tan solo un 5 por ciento de energía mediante la energía solar es una cantidad superficial que está muy por debajo de su capacidad. El país no está explotando su potencial energético con las energías renovables, no solo la energía solar sino también la mareomotriz, geotérmica y eólica. Es discutible si esta propuesta es más política que ecológica puesto que los EAU prefiere invertir en el desarrollo de la energía nuclear y el uso del carbón en vez de utilizar las energías renovables.

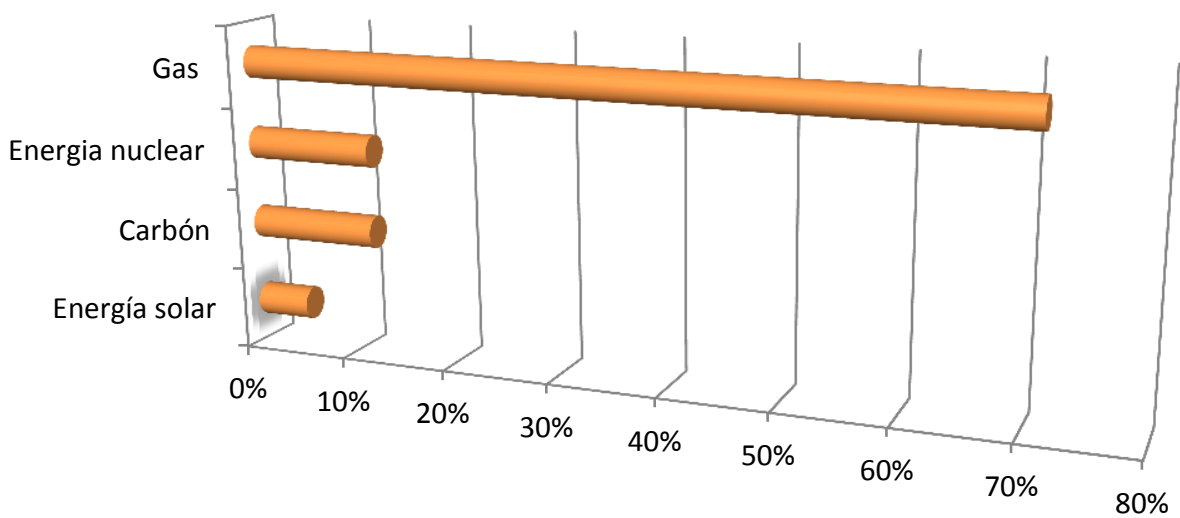

Figura 2. Diversificación de las energías en Dubai para el año 2030

\section{El agua}

Los emiratos de Abu Dabi y Dubái han crecido en población a la misma velocidad que lo han hecho su consumo de energía y agua, en particular durante los meses más cálidos del verano y muchos expertos advierten que el aumento de la demanda se está convirtiendo en insostenible. 
El agua dulce ha sido siempre un bien muy preciado en esta parte del mundo, y sigue siendo el caso a pesar de que los Emiratos Árabes Unidos ahora tenga los recursos para cumplir con su demanda de agua potable a través de la desalinización. Sin embargo los científicos advierten que los suministros de agua subterránea del país se están agotando de manera insostenible. El exceso de extracción ha causado que la tabla de agua caiga drásticamente en los últimos años.

La agricultura representa algo más de un tercio del uso del agua en general y dos tercios del agua subterránea extraída. Si no se hace nada para detener este nivel de sobre extracción, los investigadores advierten que los Emiratos Árabes Unidos podría quedarse sin agua subterránea para el año 2030 (Malek, 2015). El gobierno se apoya en el uso de la energía nuclear y en menor medida la energía solar como recursos sostenibles para la desalinización de agua de mar, sin embargo, la seguridad alimentaria y de agua basada únicamente en la producción nacional es imposible.

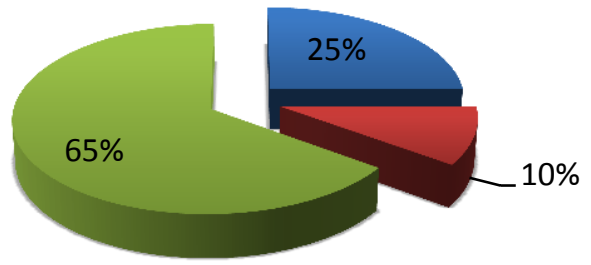

nesidencial y comercial $\quad$ Industrial $\quad$ Agricultura

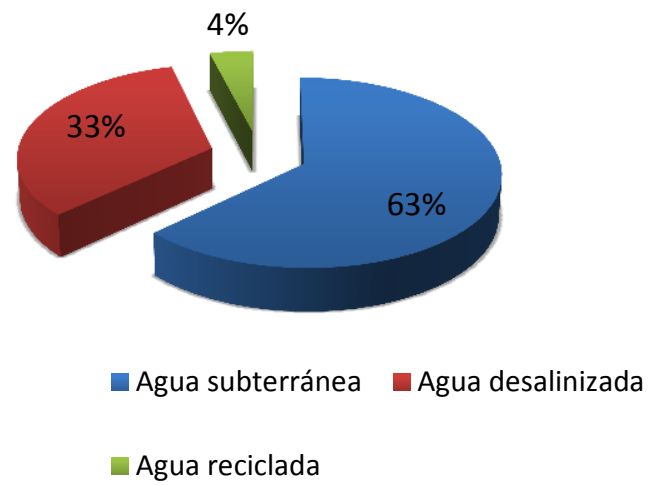

Figura 3. Gráfica de dónde se consume el agua y su lugar de procedencia.

La seguridad alimentaria implica cuatro dimensiones: la disponibilidad, que es la producción y el comercio; la accesibilidad - física y económica; el uso, nutricional y de seguridad alimentaria; y la estabilidad a través del tiempo, la sostenibilidad. Algunas soluciones para permitir que los suministros de agua subterránea se recuperen, sería principalmente dar prioridad a que se destine el agua a nivel nacional. La inversión de los EAU en las granjas de Europa, África y Asia está diseñado para disminuir la necesidad de alimentos de cosecha propia, aunque esto plantea sin duda un tema aparte, la seguridad del suministro. La seguridad del agua tiene que ser de máxima prioridad en el país. El agua para la agricultura tiene que tener prioridad sobre su uso para jardinería, pero cualquier solución debe ser multifacética e incluir una mayor recuperación de las aguas residuales mediante políticas que protejan este recurso, el uso de las plantas más adecuadas para ambientes áridos, educar y elevar el nivel de conciencia de la comunidad y sobre todo imponer prácticas agrícolas más eficientes.

En los últimos 30 años ha habido un aumento en la temperatura total de la península arábiga de aproximadamente $1,6{ }^{\circ} \mathrm{C}$ (Al Wasmi, N., 2015). El aumento de la temperatura en esta región afecta 
dramáticamente la retención de agua y crea problemas de abastecimiento, ya que cada grado de aumento de la temperatura se corresponde con un incremento del 5 por ciento en las tasas de evaporación. Debido a que una parte significativa del agua de EAU proviene de acuíferos subterráneos, la acentuación de la temperatura en los últimas décadas años incide en un aumento de casi el 7 por ciento en la evaporación del agua de los recursos hídricos subterráneos y superficiales. Aunque eso podría no parecer importante, esas fuentes de agua subterráneas disponibles se reducen aún más si se tienen en consideración las tasas de contaminación y la falta de políticas para proteger esas fuentes. Solo se necesita un mililitro de crudo para contaminar un millón de mililitros de agua.

Con una precipitación media anual de 120 milímetros, las nuevas técnicas para la producción de alimentos con menos agua tendrá que ser introducida en los Emiratos Árabes Unidos para hacer frente a su creciente población. De acuerdo con un estudio realizado por la Universidad de los Emiratos Árabes Unidos, las nuevas técnicas de riego, tales como la superficie y por aspersión han permitido al país ahorrar alrededor de un 60 por ciento de agua en comparación con los antiguos métodos aplicados, tales como inundaciones y surco. La producción de cultivos intensivos en agua tiene que cesar a favor de la introducción de invernaderos de nueva generación y otras medidas tales como la agricultura con clima inteligente, cultivos de uso eficiente del agua, el uso de aguas residuales tratada para la agricultura y buscar cultivos que toleren altas temperaturas para garantizar la sostenibilidad.

El Consumo de agua y electricidad doméstico

El incremento anual en el consumo de energía en Abu Dabi es alrededor del 8 por ciento, mientras que el agua está aumentando al 4 por ciento anual. El emirato en 2014 consumió 1.100 millones de litros de agua desalinizada. Cualquier aumento en costos de servicios públicos probablemente contribuirá a aumentar el costo de vida en el emirato, y consecuentemente en el país. Los ciudadanos pueden reducir significativamente sus facturas mediante cambios en los patrones de consumo. Una nueva legislación se ha introducido en enero de 2015 para racionalizar el consumo de energía y reducir los subsidios.

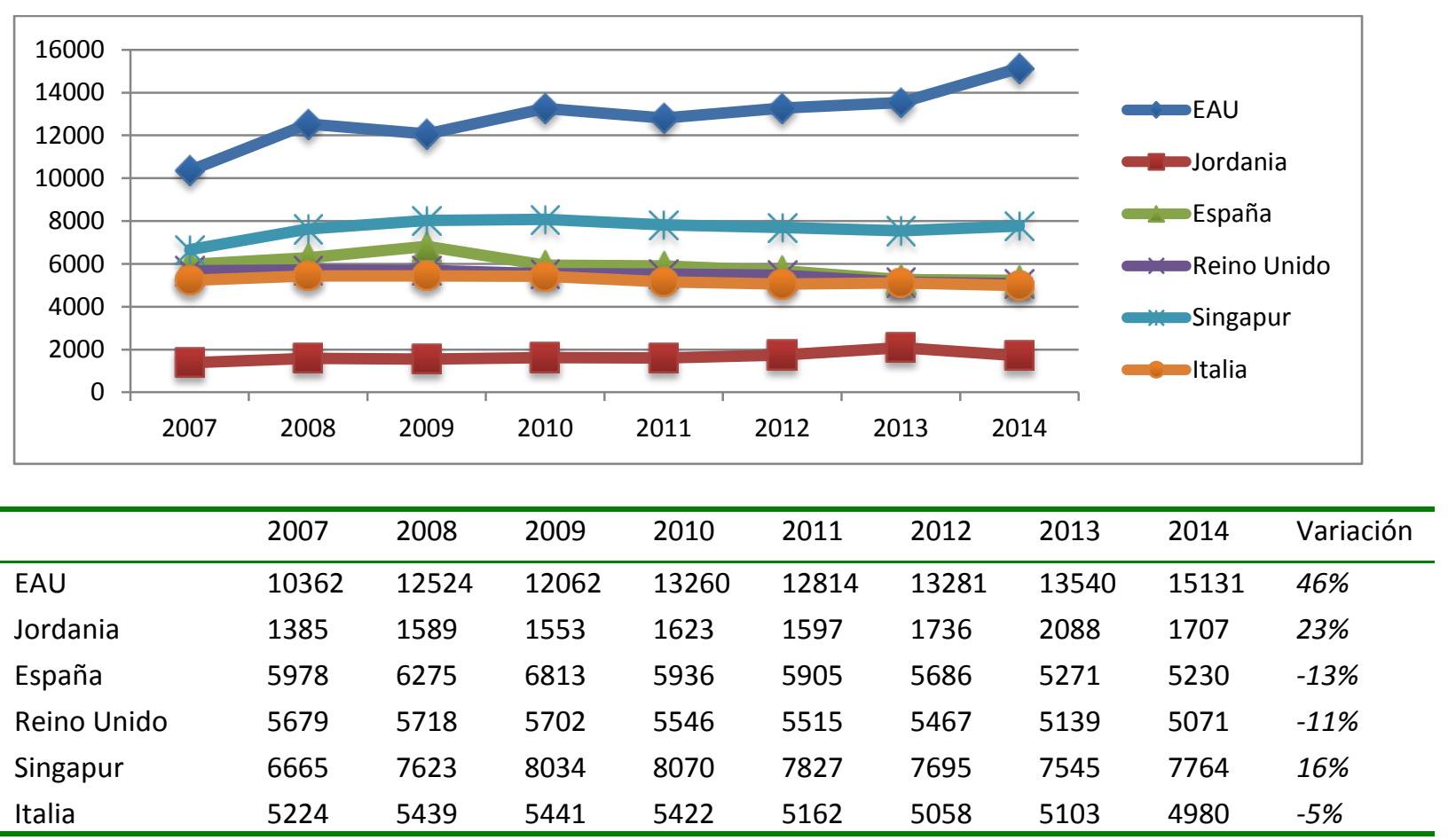

Fuente. Statista

Figura 4. Consumo eléctrico per cápita (kWh por persona) 
El coste de la electricidad y agua en EAU se incrementó en enero de 2015 pero sigue siendo mucho más barato que en países vecinos como Jordania gracias a los subsidios que los habitantes reciben y en particular los emiratíes. Además los consumidores que se comprometen a límites de consumo seguirán recibiendo subsidios extraordinarios del gobierno (Halligan, 2015).

En 2014 los subsidios de energía y de agua costaron al emirato de Abu Dabi unos 17.500 millones de dirhams. Eso es un incremento de más de un 9 por ciento respecto al año anterior, y podría elevarse a 45.000 millones en los próximos años. Sin embargo muchos ciudadanos aún siguen pensando que la electricidad es cara en el país con un tercio de los encuestados que opinan que el precio del agua y la electricidad es caro o muy caro.

Los Emiratos Árabes Unidos es el séptimo mayor productor de energía del mundo, con reservas probadas de petróleo de 97,8 mil millones de barriles, suficiente para durar más de 100 años, si se bombease en el nivel actual.

Los Emiratos Árabes Unidos tiene casi la tasa más alta de consumo de energía por persona en el mundo. Un residente de Emiratos Árabes Unidos consume 8.271 kilogramos de energía equivalente de petróleo (kgep) anual, muy por debajo de algunos de sus vecinos, como Qatar, que tiene un consumo de energía per cápita de 12.799 kgep o Kuwait con 12.204 kgep. Sin embargo, si lo comparamos con el consumo per cápita de energía en el Reino Unido y los Estados Unidos en 2014, fue de 3,254 y 7,164 kgep respectivamente, es evidente que el gobierno de EAU debe tratar de promover medidas para que sus ciudadanos sean más eficientes con la energía que consumen.

Su clima extremo necesita una gran cantidad de energía para hacer funcionar las unidades de aire acondicionado, los refrigeradores, las bombillas, los televisores, las lavadoras, los ordenadores, en fin cualquier producto electrónico imaginable en esta era tecnológica. Y como el clima seco y caliente de la tierra del desierto no se presta fácilmente a la agricultura, el país debe importar la gran mayoría de la comida, que trae otro coste energético. Es más, gran parte del agua es desalinizada, que también consume grandes cantidades de energía. Si se sigue al ritmo actual, la demanda de energía simplemente superará la oferta, simplemente no habrá suficiente para todos. La escasez provocará cortes eléctricos y una racionalización del agua, que a lo mejor son necesarios para poder concienciar a la población.

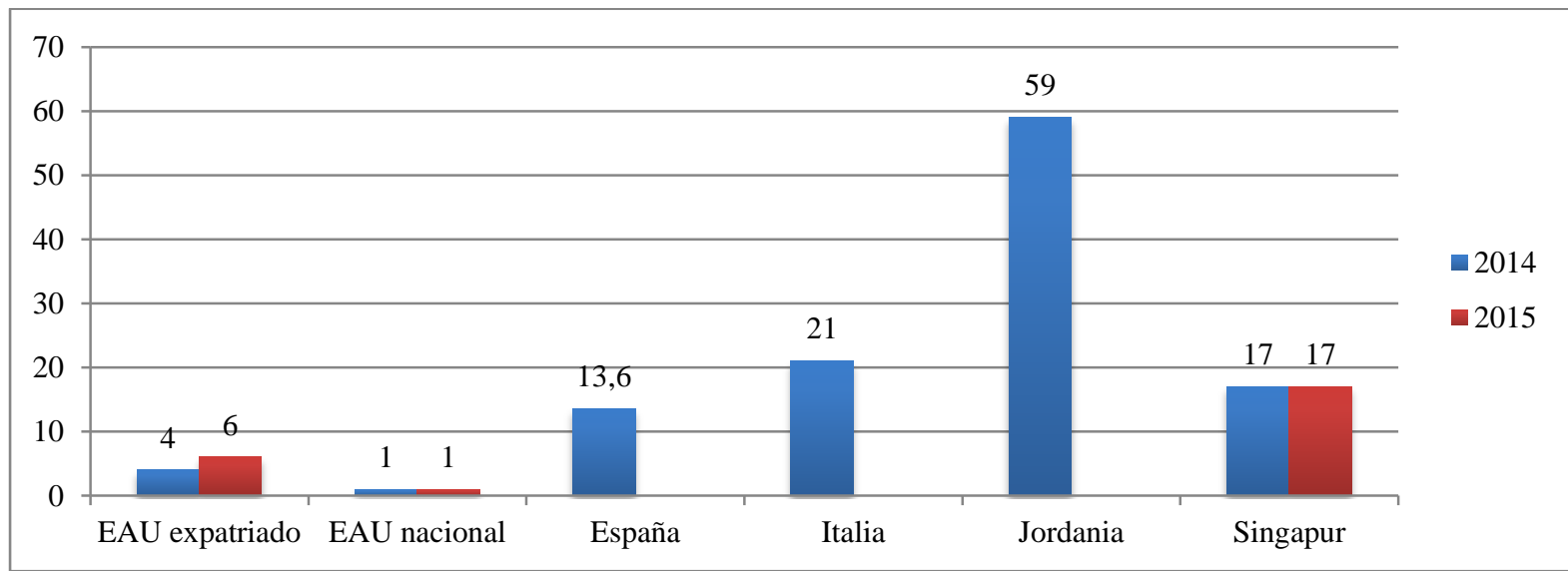

Fuente. Statista

Figura 5. Precio de la electricidad en 2014 (céntimos de USD por kWH)

Mientras que el gobierno de EAU continúe con su plan de subvencionar la electricidad, el agua y la gasolina, los ciudadanos pagarán tan solo entre un 2 y un 10 por cierto de lo que paga un residente de Jordania, país vecino y con un clima similar a los EAU (Binsal, A., 2014). Esta acción gubernamental lejos de ayudar a reducir el consumo energético del país, fomenta una actitud irresponsable hacia el consumo de estos recursos no renovables. Asimismo, las subvenciones son una carga para el gobierno que conllevan un importante coste adicional en las arcas públicas, este coste podría llegar a 45.000 millones de dírhams únicamente en el Emirato de Abu Dabi en los próximos años. 
El consumo interno de petróleo y gas está aumentando rápidamente. Los Emiratos Árabes Unidos que fue durante décadas un exportador no solo de petróleo sino también de gas, es ahora un importador neto de gas natural. El crecimiento de la demanda ha obligado al gobierno a considerar la diversificación de sus opciones de importación de gas, incluido el gas natural licuado (GNL). La seguridad energética se ha convertido en crucial para el desarrollo sostenible para todos los países, especialmente los Emiratos Árabes Unidos, la segunda mayor economía árabe.

Al mismo tiempo, el consumo de energía se suma al problema mundial del calentamiento global. Como la mayor parte de la energía proviene de la quema de combustibles fósiles, cuanto más se consume y se convierte en energía, más dióxido de carbono (CO2) se emite. El aumento de los niveles de $\mathrm{CO} 2$ conducen a más calor atrapado en la atmósfera y por lo tanto contribuyen directamente al aumento de las temperaturas. La temperatura ha aumentado lo suficiente como para tener una serie de impactos drásticos. Y sólo puede empeorar si el gobierno no propone claras directrices a seguir. Un desarrollo sostenible no será posible si tan solo un $8 \%$ de los habitantes del país toman conciencia de sus propias acciones y se responsabilizan de la enorme huella ambiental que lleva carreando el país desde hace 10 años, es fácil echar la culpa al gobierno y a las grandes compañías y multinacionales por el lastre ambiental.

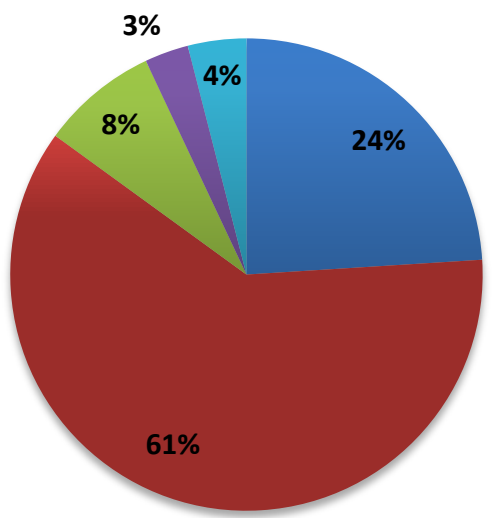

Gobierno

- Grades multinacionales

- Población

- Medios de comunicación

- Otros

Figura 6. Los ciudadanos opinan sobre el principal responsable de la huella ambiental

Los Emiratos Árabes Unidos utiliza 481 toneladas equivalentes de petróleo para producir 1 millón de dólares de su PIB. Por el contrario, Noruega sólo requiere 172 toneladas equivalentes de petróleo. Los esfuerzos de eficiencia no sólo deben centrarse en la reducción del consumo en términos absolutos, sino también en hacer al país competitivo con otros países, se tiene que utilizar menos energía para hacer crecer la economía.

Por ello, EAU está desarrollando plantas de energía nuclear para una mejor combinación de sus fuentes de energía y la reducción de su dependencia de los combustibles fósiles. Se espera que para el año 2020 la energía nuclear pueda abastecer hasta un 25 por ciento de las necesidades eléctricas del país (Rahman, 2013).

Además, el Gobierno de los Emiratos Árabes Unidos está llevando a cabo una política racional para cumplir los objetivos de desarrollo sostenible adoptadas por el propio país. Esta política se centra en tres temas principales: en primer lugar, el desarrollo de la industria petrolera para reducir el impacto negativo del sector. En segundo lugar, abordar todas las formas de uso excesivo de los recursos energéticos, con el fin de minimizar el impacto negativo del consumo excesivo en la calidad del aire y el cambio climático, y en tercer lugar, la diversificación de las fuentes de energía para reducir la presión sobre las reservas estratégicas de petróleo con el fin de garantizar la longevidad de esta fuente de riqueza nacional no renovable. 
Sustainability, Agri, Food and Environmental Research 4(1), 2016: 22-32

ISSN: 0719-3726

\section{Referencias}

Al Wasmi N., 2015. Water data adds to prediction problems. The National UAE.

Binsal A., 2014. Abu Dhabi revises water and electricity tariff. Gulf News. Dubái, UAE.

Carvalho S. \& El Dahan M., 2015. Dubai taps cheaper solar to raise renewable energy target". Reuters Africa. Abu Dabi, EAU.

Centro de Estadística de Abu Dabi., 2011. Estadísticas sobre residuos en el Emirato de Abu Dabi. Agencia Europea de Medio Ambiente. Abu Dabi, Emiratos Árabes Unidos. Disponible en: http://www.ead.ae/wpcontent/uploads/2014/04/Water Guide English.pdf Consultado en 13/07/2015 a las 11:33

Ecoembes. (2014). El reciclaje en datos. Madrid, España. Disponible en: https://www.ecoembes.com/es/ciudadanos/envases-y-proceso-reciclaje/reciclaje-en-datos Consultado en 18/07/2015 a las 20:00

Emirates News Agency, Wam, 2014a. UAE needs to curtail domestic energy consumption habits: Energy Minister. Emirates247.com.

Emirates News Agency, Wam, 2014b. Consejo Ejecutivo de Abu Dhabi. Emirates247.com.

Environment Agency en cooperación con The Center of Waste Management en Abu Dabi, 2013. Towards integrated waste management in Abu Dhabi. Abu Dabi. Disponible en: http://www.ead.ae/wpcontent/uploads/2014/03/Waste-PB-Eng.pdf Consultado en 21/07/2015 a las 12:32

Environment Agency, 2014. Protecting O/ur Shared Resource. Sustainable Water use for organisations. Abu Dabi, EAU.

Halligan, N., 2015. Residentes sufren el aumento del coste de la electricidad y agua". Arabian Business. EAU. Marzo.

Heroes of the UAE., 2015. A sustainable UAE, every child's right. Environmental Agency. Abu Dabi.. Instituto de Recursos Mundiales. Disponible en: www.wri.org Consultado en 21/07/2015 a las 21:18 Maktoob News., 2015. Aquifers face depletion in 50 years. Al Nisr Publishing LLC. EAU.

Malek C., 2015. Estudio de la Universidad de EAU recomienda priorizar el agua y cambiar las prácticas agrícolas. Gulf News. Dubái, UAE.

Ojeda A., 2011, Medioambiente en Emiratos Árabes Unidos". Extenda. Agencia Andaluza de Promoción Exterior. Dubái. EAU.

Rahman S., 2013. “UAE's per capita energy consumption among the highest. Gulf News. Dubái, EAU.

Saadi D., 2014. Dubái invierte 50.000 millones de dírhams para reducir el consumo de energía”. The National. Abu Dabi, EAU.

Simpson C., 2013. UAE's largest power and desalination plant opens in Jebel Ali. EL periódico The National. UAE News, Dubái.

Statista. Consumo eléctrico per cápita. Disponible: http://www.statista.com/statistics/263492/electricityprices-in-selected-countries/ Consultado en 24/07/2015 a las 11:15

Statista. Electricity prices. Disponible en: http://www.statista.com/statistics/263492/electricity-prices-inselected-countries/ Consultado en 15/07/15 a las 18:35 